\title{
Lorentz Transmission Electron Microscopy for Imaging Magnetic Fields from a Perpendicular Ferromagnetic Stripe Domain Thin Film
}

\author{
Taeho Roy $\mathrm{Kim}^{1}$, Olav Hellwig ${ }^{2}$ and Robert Sinclair ${ }^{1}$ \\ 1. Department of Materials Science and Engineering, Stanford University, Stanford, CA 94305-4034. \\ 2. San Jose Research Center, HGST, a Western Digital Company, 3403 Yerba Buena Road, San Jose, \\ CA 95135.
}

Perpendicular magnetic thin films, widely used high density storage media, need further improvement in order to meet the increasing demand for data storage. To study them, it is important to understand both the microstructure and magnetic properties. One useful technique to study such films is magnetic force microscopy (MFM) [1]. However, MFM can only detect magnetic information of a material with no structural information such as grain structure.

Here we apply Lorentz Transmission Electron Microscopy (LTEM) to this problem. This has been used successfully to study magnetic films with in-plane magnetic domains [2], but here we show the capability of LTEM for perpendicular ferromagnetic stripe domain thin films. We chose the Fresnel mode among various techniques in LTEM [3] because a simple experimental procedure of Lorentz lens defocus provides qualitative information on magnetization. The Lorentz force from a magnetic material deflects the electron beam and this deflection gives rise to contrast in the image. The results suggest that plan view images are comparable to MFM images shown in a previous study by Hellwig et al [4]. Furthermore, cross-sectional images allow observation of the magnetic fields emerging from the thin film that cannot be observed by MFM.

We studied two Co/Pd multilayer films grown on a Si substrate with perpendicular ferromagnetic stripe domains. The number of Co/Pd layers of the cross-sectional sample was about 2.5 times more than that of the plan view sample, but our purpose of imaging magnetic fields is not affected. The detail of the $\mathrm{Co} / \mathrm{Pd}$ multilayer film structure, fabrication method and stripe domain formation process are described elsewhere [4]. Both plan view and cross-sectional TEM specimens were prepared using a conventional method of grinding, polishing and ion milling. Magnetic imaging was then carried out on an FEI Titan 80-300 TEM at the Stanford Nanocharacterization Laboratory. The TEM was operated at $300 \mathrm{kV}$ in Lorentz mode with the objective lens off to provide an environment free of magnetic fields. Images were acquired at large defoci up to about $2 \mathrm{~mm}$ and then were digitally processed with histogram equalization to show better the magnetic contrast.

Plan view images are shown in Figure 1. The in-focus image shows a low magnification view of the grain structure and the defocused image shows stripe patterns due to magnetic domain contrast. The magnetic field components perpendicular to the electron beam at domain boundaries cause bright or dark contrast. In Figure 2, cross-sectional images are shown. The in-focus image shows that portions of the film are unintentionally etched away during TEM specimen preparation. The defocussed image shows magnetic domain contrast outside the film, in free space, which is not present where the magnetic film has been removed. There are bright lines due to the magnetic domain structure. Interestingly, these features are visible in the vacuum extending up to eight times the film thickness and their contrast weakens as the film becomes thinner. 
The study demonstrates the effectiveness of the LTEM for studying perpendicular ferromagnetic stripe domain thin films. Both plan view and cross-sectional images reveal the presence of magnetic domains. However the correlation with the grain structure has still not been achieved.

[1] M. Alberecht et al, Appl. Phys. Lett. 81 (2002), p. 2875-2877.

[2] K Tang et al, IEEE Trans. Magn. 32 (1996), p. 4130-4132.

[3] C A Ross et al in "Wiley Encyclopedia of Electrical and Electronics Engineering: Magnetic Media, Imaging”, J. Webster, (John Wiley \& Sons, Inc., Hoboken) (1998), p.1-6.

[4] O Hellwig et al, J. Magn. Magn. Mater. 319 (2007), p. 13-55.

[5] The authors thank Western Digital Inc. for financial support for this study.
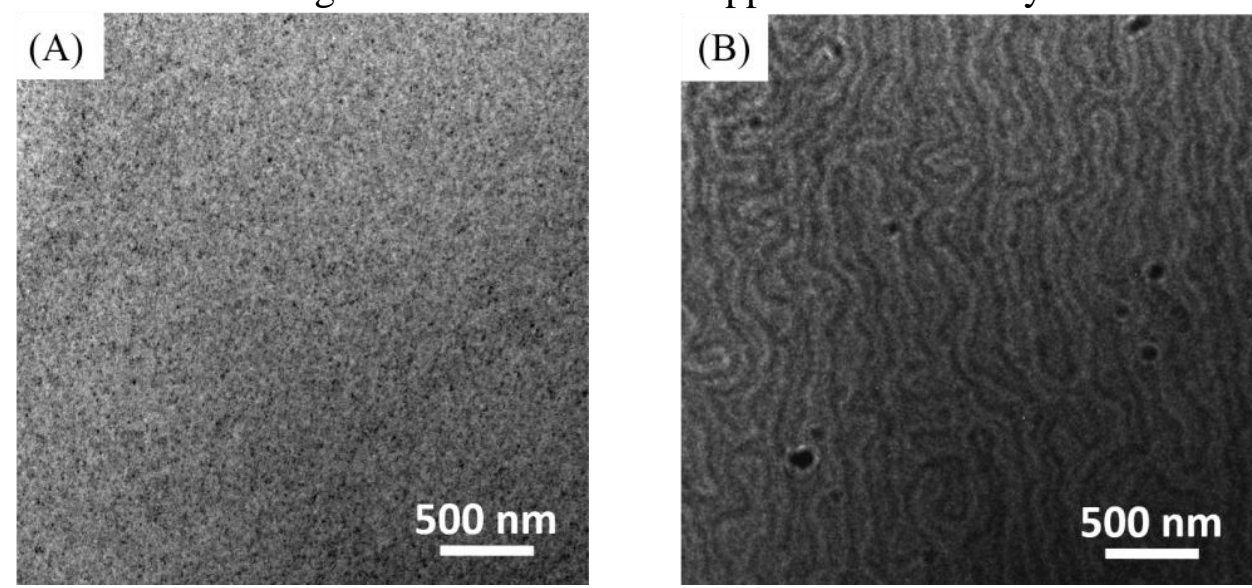

Figure 1. Plan view images of a Co/Pd multilayer film using LTEM. (A) Low magnification in-focus image showing the $20 \mathrm{~nm}$ level grain structure. (B) Under-focused (about $750 \mu \mathrm{m}$ ) image of the same region with stripe patterns representing magnetic domains.
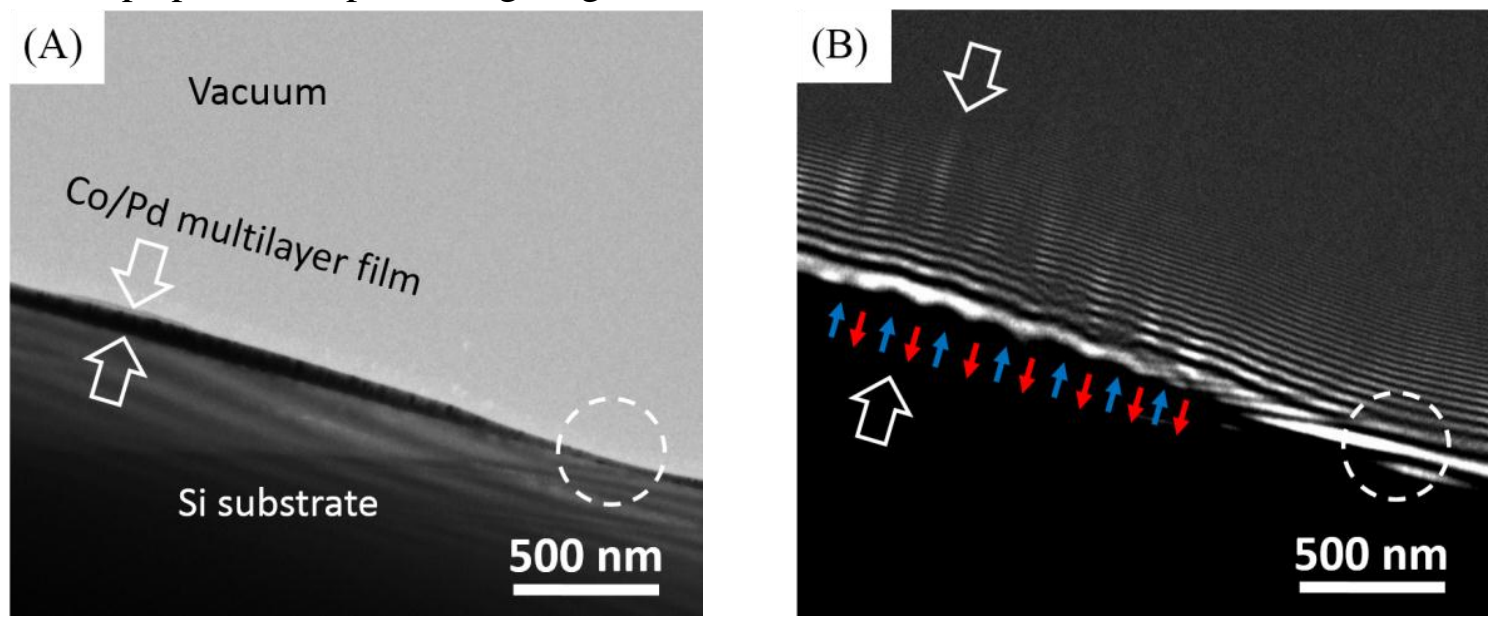

Figure 2. Cross-sectional images. (A) Focused image showing the film on a Si substrate. Arrows indicate the full stack region of the film with a thin layer of epoxy from the TEM specimen preparation on top. The dashed circle indicates the region where most of the film is etched away during the TEM specimen preparation. (B) Large under-focused (about $2 \mathrm{~mm}$ ) image showing bright regions in a dark background due to magnetic fields from the film. The longest bright region implying strongest magnetic strength is located where the magnetic thin film is still remaining (the arrows from (A) are indicating the same location). In the dashed circle, no magnetic contrast is observed as expected. Small arrows indicate the possible location of perpendicular magnetic domains and their expected field directions in the film. 